\title{
Parámetros del crecimiento físico de niños que viven a moderada altitud.
}

\author{
Parameters of physical growth of children living at moderate altitude. \\ Marco Cossio-Bolaños ${ }^{1}$, Pascual Figueroa ${ }^{2}$, Wilbert Juvenal Cossio-Bolaños ${ }^{3}$, Evandro Lázari ${ }^{4}$, Miguel \\ Arruda ${ }^{5}$.
}

\section{RESUMEN}

El desarrollo del crecimiento físico depende del bagaje genético, nutricional, nivel socio-económico, enfermedades, influencias ambientales, tendencia secular, actividad física y el nivel de altitud. Objetivo: Determinar los parámetros del crecimiento físico de niños que viven a moderada altitud en función del género y en relación a los patrones referenciales del CDC y NHANAS-USA. Material y métodos: Fueron seleccionados 795 niños de condición socioeconómica media de manera probabilística (estratificado), siendo 394 varones y 401 damas de escuelas públicas del área urbana de la ciudad de Arequipa-Perú $(2320 \mathrm{msnm})$. Se evaluaron las variables antropométricas peso corporal ( $\mathrm{kg}$ ), estatura $(\mathrm{m})$, pliegue tricipital $(\mathrm{mm})$ y circunferencia del brazo relajado (cm). Resultados: Se encontró valores similares en el peso corporal $(\mathrm{p}>0,005)$ y valores inferiores en estatura $(\mathrm{p}<0,005)$ en niños de ambos géneros en relación con la referencia del CDC. En el pliegue tricipital, se observó valores relativamente similares en ambos géneros, al comparar con la referencia del National Health and Nutrition Examination Survey (NHANES)-USA. Los valores de la circunferencia del brazo según percentiles fue menor en ambos géneros en comparación con la referencia NHANES-USA. Conclusiones: Los resultados sugieren atraso en el crecimiento lineal que podría estar relacionada con la altitud y con una pobre reserva de proteína observada en la circunferencia del brazo.

PALABRAS CLAVE: Crecimiento, altitud, niño, antropometría. (Fuente: DeCS BIREME).

\section{SUMMARY}

The development of physical growth depends on genetic background, nutritional, socioeconomic conditions, environmental, secular trend, physical activity and level of altitude. Objective: To determine the parameters of physical growth of children living at moderate altitude according gender and compared with reference patterns CDC and NHANAS-USA. Methods: We selected 795 children from socio-economic status mean, by probabilistic sampling (stratified); 394 males and 401 women from public school of urban area of Arequipa city, Peru (2320msnm). We evaluated body weight $(\mathrm{kg})$, height $(\mathrm{m})$, triceps skinfold $(\mathrm{mm})$ and relaxed arm circumference $(\mathrm{cm})$. Results:

1 Doctor en Ciencias del Deporte. Faculdade de Educação Física Universidade Estadual de Campinas. Sao Paulo, Brasil.

2 Doctor en Ciencias de la Computación, Instituto de Computación de la Universidade Estadual de Campinas Sao Paulo, Brasil.

3 Especialista en Estadística e investigación, Facultad de Medicina Alberto Hurtado, Universidad Peruana Cayetano Heredia. Jefe de la Sanidad Naval de Ventanilla, Marina de Guerra del Perú. Callao, Perú.

4 Msc en Ciencias del deporte, Faculdade de Educação Física. Universidade Estadual de Campinas. Sao Paulo, Brasil.

5 Doctor en Ciencias del Deporte. Prof. Titular de la Faculdade de Educação Física de la Universidade Estadual de Campinas. Sao Paulo, Brasil. 
We found similar values of body weight $(p>0.005)$ and lower values of stature $(p<0.005)$ in both genders when compared with CDC reference. In the triceps skinfold, there was similar values in both genders when compared with the reference National Health and Nutrition Examination Survey (NHANES)-USA. Values of arm circumference was lower in both gender in comparison with reference NHANES-USA. Conclusions: The results suggest delay in linear growth may be related to altitude and with poor reserves of protein observed in arm circumference.

KEYWORDS: Growth, altitude, child, anthropometry (Source: MeSH NLM).

\section{INTRODUCCIÓN}

El crecimiento humano es un fenómeno complejo que es influenciado por factores intrínsecos y extrínsecos (1). Los factores intrínsecos están concentrados en los sistemas neuro-endócrinos, en el esqueleto y en la rigidez de los órganos efectores terminales y las células; y los factores extrínsecos abarcan una extensa gama de características ambientales.

El crecimiento depende del bagaje genético, nutricional, nivel socio-económico, dolencias, influencias ambientales, tendencia secular, actividad física (2) y el nivel de altitud (3-6). Su valoración puede realizarse a través de la técnica antropométrica, siendo la más conveniente, simple, económica y es un método no-invasivo (7). Esta técnica permite caracterizar patrones de crecimiento y de la composición corporal (8) de niños, jóvenes y adultos. Su medición se puede realizar con medidas simples como la estatura, peso corporal, circunferencias, pliegues cutáneos y diámetros óseos (9).

Estas herramientas son aceptadas para el control de la salud y evaluación del estado nutricional de niños (10). A su vez, permite evaluar el impacto de los factores ambientales y genéticos en la adaptación biológica de las poblaciones humanas, tanto del nivel mar, como en la altitud.

Es ampliamente conocido que los estudios referenciales como los de la Organización Mundial de la salud (OMS), del Centro para el Control y la Prevención de Enfermedades (CDC), fueron desarrollados en base a poblaciones sobre el nivel del mar, por lo que no podrían ser aceptados como parámetros de referencia del crecimiento en poblaciones que viven en la altura (11), sobre todo, si el 6\% de la población mundial vive sobre los $1500 \mathrm{msnm}$ (12) y en el Perú el 33\% de la población, vive arriba de los $3000 \mathrm{msnm}$ (13).

En este sentido, la utilización de los parámetros de referencia mencionados, en poblaciones que habitan en la altura podría ocasionar sesgo en la interpretación de los resultados. Pero, algunos países como Perú no cuentan con patrones referenciales que permitan valorar el crecimiento físico y el estado nutricional de niños y adolescentes, lo que obliga a usar parámetros internacionales desarrollados en poblaciones de nivel del mar.

El objetivo del estudio fue determinar los parámetros del crecimiento físico de niños que viven a moderada altitud en función del género $\mathrm{y}$ en relación con los patrones referenciales CDC y del National Health and Nutrition Examination Survey (NHANAS)-USA.

\section{MATERIAL Y MÉTODOS}

Estudio descriptivo y trasversal, realizado durante el 2002, en niños escolares de Arequipa, Perú, ubicada a 2320 msnm.

\section{Muestra}

El universo estuvo conformado por 5627 escolares de colegios estatales de zonas urbanas 
del distrito de José Luis Bustamante y Rivero de Arequipa, Perú. El tamaño de la muestra se determinó por selección probabilística de tipo estratificado. Se seleccionaron 795 niños de ambos sexos (394 niños y 401 niñas). Se incluyeron en el estudio a todos los niños que pertenecían a la condición socioeconómica media y los que vivían de forma permanente en la ciudad de Arequipa y en áreas urbanas.

Para determinar la condición socioeconómica media, se aplicó un cuestionario propuesto por Cossio-Bolaños (14), en el que se incluyen 7 preguntas, los que permitieron identificar a los escolares de condición socioeconómica media por medio de una escala que va de 36 a 59 puntos. Valores inferiores indican condición baja y valores superiores condición alta.

\section{Técnicas e instrumentos}

Las variables antropométricas fueron evaluadas siguiendo las normas y sugerencias estandarizadas por el "International Working Group of Kineanthropometry" descrita por Ross y Marfell-Jones (15). Todas las variables antropométricas fueron evaluadas en el lado derecho para ambos sexos:

Masa corporal (kg): se utilizó una balanza digital con una precisión de $(200 \mathrm{~g})$ de marca Tanita con una escala de (0 a $150 \mathrm{~kg})$.

Estatura (m): fue evaluada utilizando un estadiómetro de aluminio graduada en milímetros de marca Seca, presentando una escala de $(0-2,50 \mathrm{~m})$.

Pliegue cutáneo tricipital: Se evaluó la doble capa de piel de la región tricipital $(\mathrm{mm})$, utilizando un compás de pliegues cutáneos de Marca Harpenden con una presión constante de $\left(10 \mathrm{~g} / \mathrm{mm}^{2}\right)$.

Circunferencia del brazo: Se evaluó la circunferencia media del brazo relajado $(\mathrm{cm})$ utilizando una cinta métrica de nylon Seca milimetrada con una precisión de $(0,1 \mathrm{~cm})$. Aspectos éticos

El estudio contó con la respectiva aprobación del comité de ética del Instituto del Deporte Universitario IDUNSA de la Universidad Nacional de San Agustín de Arequipa. Todos los responsables y tutores de los niños llenaron y firmaron la ficha de consentimiento libre y aclarado para autorizar las evaluaciones antropométricas.

\section{Normas referenciales utilizadas}

Se utilizó las normas del Centro para el Control y la Prevención de Enfermedades (CDC) (16) para el peso y estatura. Para el pliegue tricipital y la circunferencia del brazo relajado las normas de la Encuesta Nacional de Salud y Nutrición NHANAS-USA propuestas por Frisancho (17). Los resultados se compararon a partir del p5, p10, p25, p50, p75, p90 y p95 adoptados como puntos de corte por cada norma, respectivamente.

\section{Confiabilidad de las medidas}

Todas las variables antropométricas mostraron un error técnico de medida (ETM) intraevaluador inferiores al $2 \%$ y un coeficiente de reproductibilidad de $(\mathrm{r}=0,98)$.

\section{Análisis estadístico}

La distribución normal de los datos se verificó por medio de la prueba de Kolmogorov-Smirnov. Los resultados fueron analizados a través de la estadística descriptiva con media aritmética, desviación estándar y percentiles. Se utilizó como puntos de corte p5, p10, p25, p50, p75, p90 y p95. Para determinar las diferencias significativas entre ambos sexos se utilizó la prueba t de Student para muestras independientes y la prueba de especificidad de Tukey con una probabilidad de $(p<0,005)$. La comparación entre edades de un mismo género (intra-grupos) se utilizó ANOVA de una vía con una probabilidad de $(\mathrm{p}<0,005)$. Para comparar los valores del peso y la estatura del estudio y la referencia se utilizó la prueba $t$ 
de student para muestras apareadas $(\mathrm{p}<0,005)$. La comparación del pliegue tricipital y la circunferencia del brazo entre la referencia y el estudio, según percentiles se realizó por medio de la fracción: $100 \log$ (percentil de la referencia/percentil del estudio).

\section{RESULTADOS}

En la tabla 1 se muestra los resultados de las variables antropométricas utilizadas en el estudio. Todos los valores fueron expresados como promedio y desviación estándar para describir la población estudiada. Los resultados muestran que las niñas presentan valores más altos en los pliegues cutáneos en relación a los varones.

Las medias, desviación estándar y los valores según los percentiles de la masa corporal de los niños de ambos sexos se pueden observar en la tabla 2. Los valores muestran una tendencia ascendente con la edad en ambos sexos. Ala vez, se observa que las niñas a partir de los 9 años empiezan a presentar mayor masa corporal que los niños (Figura 1). Así mismo, al comparar con la referencia del CDC, no se encontró diferencias significativas en ambos géneros, salvo a los seis años en las niñas. (Figura 2).

Los valores según percentiles y los valores promedios de la estatura, se muestran en la tabla 3. Se encontró que las niñas tuvieron mayor estatura en las edades de 6, 7 y 9 años en comparación con los varones de la misma edad. En ambos géneros, podemos observar una tendencia ascendente con la edad (Figura 1). Comparando con los valores del CDC en el p50, los valores de nuestro estudio fueron menores en ambos géneros, con excepción en las niñas a los 9 años y en los niños a los 6 años (Figura 2).

En la tabla 4, se muestra la diferencia entre

Tabla 1. Características de la población estudiada.

\begin{tabular}{|c|c|c|c|c|c|}
\hline Edad (años) & & Masa Corporal (kg) & Estatura (m) & $\begin{array}{l}\text { Pliegue Tricipital } \\
\text { (mm) }\end{array}$ & $\begin{array}{c}\text { Circunferencia } \\
\text { Brazo }(\mathrm{cm})\end{array}$ \\
\hline \multirow{2}{*}{6} & Niños & $21,81 \pm 2,24$ & $1,14 \pm 0,05$ & $9,01 \pm 1,99$ & $16,70 \pm 1,08$ \\
\hline & Niñas & $22,48 \pm 2,62 *$ & $1,19 \pm 0,05$ & $10,53 \pm 2,17 *$ & $17,00 \pm 1,10$ \\
\hline \multirow{2}{*}{7} & Niños & $22,91 \pm 3,92$ & $1,18 \pm 0,05$ & $9,03 \pm 2,70$ & $17,23 \pm 1,37$ \\
\hline & Niñas & $24,62 \pm 3,45^{*}$ & $1,23 \pm 0,04$ & $11,38 \pm 2,96^{*}$ & $17,40 \pm 1,51$ \\
\hline \multirow{2}{*}{8} & Niños & $25,88 \pm 3,55$ & $1,25 \pm 0,05$ & $9,29 \pm 2,50$ & $17,49 \pm 1,43$ \\
\hline & Niñas & $26,57 \pm 3,04^{*}$ & $1,26 \pm 0,06$ & $12,09 \pm 2,08^{*}$ & $17,64 \pm 1,08$ \\
\hline \multirow{2}{*}{9} & Niños & $29,38 \pm 3,80$ & $1,28 \pm 0,04$ & $9,55 \pm 3,10$ & $18,07 \pm 1,27$ \\
\hline & Niñas & $30,38 \pm 4,62 *$ & $1,32 \pm 0,06$ & $13,10 \pm 2,09^{*}$ & $18,59 \pm 1,81$ \\
\hline \multirow{2}{*}{10} & Niños & $33,25 \pm 4,05$ & $1,36 \pm 0,07$ & $10,81 \pm 2,22$ & $19,03 \pm 1,06$ \\
\hline & Niñas & $37,20 \pm 5,37^{*}$ & $1,40 \pm 0,06$ & $13,11 \pm 3,15^{*}$ & $19,54 \pm 1,84$ \\
\hline \multirow{2}{*}{11} & Niños & $37,01 \pm 4,83$ & $1,43 \pm 0,05$ & $11,58 \pm 2,68$ & $19,91 \pm 2,29$ \\
\hline & Niñas & $38,92 \pm 5,96^{*}$ & $1,44 \pm 0,05$ & $13,56 \pm 2,91 *$ & $20,45 \pm 2,50$ \\
\hline
\end{tabular}

Medias \pm Desviación estándar

* Diferencias en relación a los niños, ( ) desviación estándar, 
Tabla 2. Valores medios (X), desviación estándar (DE) y según percentiles de la masa corporal (kg).

\begin{tabular}{|c|c|c|c|c|c|c|c|c|c|c|}
\hline Edad & $\mathbf{P 5}^{\circ}$ & $\mathrm{P}_{10}^{\circ}$ & $\mathbf{P 2 5}^{\circ}$ & $\mathrm{P50}^{\circ}$ & $\mathbf{P}^{75^{\circ}}$ & $\mathrm{P90}^{\circ}$ & $\mathbf{P 9 5}^{\circ}$ & $\mathbf{X}$ & DE & $\mathbf{p}$ \\
\hline \multicolumn{11}{|c|}{ Niños $(n=394)$} \\
\hline 6 & 18,00 & 19,00 & 20,00 & 22,00 & 23,00 & 25,00 & 25,25 & 21,88 & 2,38 & 0,06 \\
\hline 7 & 18,00 & 19,00 & 20,00 & 24,00 & 25,00 & 25,00 & 30,90 & 22,91 & 3,92 & 0,81 \\
\hline 8 & 20,00 & 21,00 & 24,00 & 25,50 & 28,00 & 31,00 & 31,65 & 25,88 & 3,55 & 0,06 \\
\hline 9 & 20,00 & 24,00 & 27,00 & 30,00 & 32,00 & 34,80 & 35,00 & $29,38 *$ & 3,80 & 0,08 \\
\hline 10 & 25,00 & 27,00 & 31,00 & 33,00 & 36,00 & 38,00 & 39,00 & $33,25^{*}$ & 4,05 & 0,66 \\
\hline 11 & 29,00 & 31,00 & 33,50 & 36,00 & 41,00 & 44,00 & 45,00 & $37,01 *$ & 4,83 & 0,15 \\
\hline \multicolumn{11}{|c|}{ Niñas $(n=401)$} \\
\hline 6 & 17,50 & 19,00 & 21,00 & 23,00 & 24,00 & 25,10 & 27,05 & 22,48 & 2,62 & 0,06 \\
\hline 7 & 19,00 & 21,00 & 22,00 & 24,00 & 27,00 & 29,00 & 30,00 & 24,62 & 3,45 & 0,39 \\
\hline 8 & 19,00 & 24,00 & 25,00 & 27,00 & 29,00 & 29,00 & 30,90 & 26,57 & 3,04 & 0,05 \\
\hline 9 & 24,00 & 25,00 & 26,00 & 29,00 & 35,00 & 36,00 & 38,00 & 30,38 & 4,62 & 0,28 \\
\hline 10 & 25,00 & 29,40 & 31,00 & 36,00 & 43,00 & 43,00 & 44,80 & 36,64 & 5,47 & 0,10 \\
\hline 11 & 29,38 & 30,00 & 35,00 & 38,00 & 43,00 & 46,00 & 50,00 & 38,91 & 5,98 & 0,54 \\
\hline
\end{tabular}

*: Diferencia entre géneros ( $\mathrm{p}<0,05), \mathrm{p}=$ NS respecto a los valores del CDC (en el percentil 50).

Tabla 3. Valores medios (X), desviación estándar (DE) y según percentiles de la estatura (m).

\begin{tabular}{|c|c|c|c|c|c|c|c|c|c|c|}
\hline Edad & $\mathbf{P 5}^{\circ}$ & $\mathbf{P}^{10} 0^{\circ}$ & $\mathbf{P 2 5}^{\circ}$ & $\mathrm{P50}^{\circ}$ & $\mathbf{P}^{75^{\circ}}$ & $\mathrm{P}^{9} 0^{\circ}$ & $\mathrm{P95}^{\circ}$ & $\mathbf{X}$ & DE & $\mathbf{P}$ \\
\hline \multicolumn{11}{|c|}{ Niños $(n=394)$} \\
\hline 6 & 1,07 & 1,08 & 1,11 & 1,14 & 1,16 & 1,23 & 1,26 & $1,14^{*}$ & 0,05 & NS \\
\hline 7 & 1,09 & 1,12 & 1,13 & 1,17 & 1,2 & 1,24 & 1,26 & $1,18^{*}$ & 0,05 & 0,05 \\
\hline 8 & 1,13 & 1,20 & 1,22 & 1,24 & 1,28 & 1,32 & 1,33 & 1,25 & 0,05 & 0,05 \\
\hline 9 & 1,19 & 1,23 & 1,24 & 1,27 & 1,31 & 1,34 & 1,34 & $1,28^{*}$ & 0,04 & 0,05 \\
\hline 10 & 1,24 & 1,27 & 1,32 & 1,36 & 1,41 & 1,45 & 1,46 & 1,36 & 0,07 & 0,05 \\
\hline 11 & 1,31 & 1,35 & 1,40 & 1,41 & 1,47 & 1,50 & 1,51 & 1,43 & 0,06 & 0,05 \\
\hline \multicolumn{11}{|c|}{ Niñas $(n=401)$} \\
\hline 6 & 1,09 & 1,12 & 1,15 & 1,20 & 1,22 & 1,26 & 1,26 & 1,19 & 0,05 & 0,05 \\
\hline 7 & 1,15 & 1,18 & 1,19 & 1,22 & 1,27 & 1,27 & 1,3 & 1,23 & 0,04 & 0,05 \\
\hline 8 & 1,15 & 1,18 & 1,21 & 1,25 & 1,31 & 1,33 & 1,39 & 1,26 & 0,06 & 0,05 \\
\hline 9 & 1,17 & 1,24 & 1,27 & 1,34 & 1,37 & 1,38 & 1,39 & 1,32 & 0,06 & NS \\
\hline 10 & 1,30 & 1,32 & 1,38 & 1,39 & 1,46 & 1,47 & 1,49 & 1,40 & 0,06 & 0,05 \\
\hline 11 & 1,33 & 1,39 & 1,40 & 1,43 & 1,48 & 1,50 & 1,54 & 1,44 & 0,05 & 0,05 \\
\hline
\end{tabular}

*: Diferencia entre género $(\mathrm{p}<0,05), \mathrm{p}=$ diferencia con respecto a los valores del CDC (en el percentil 50). 

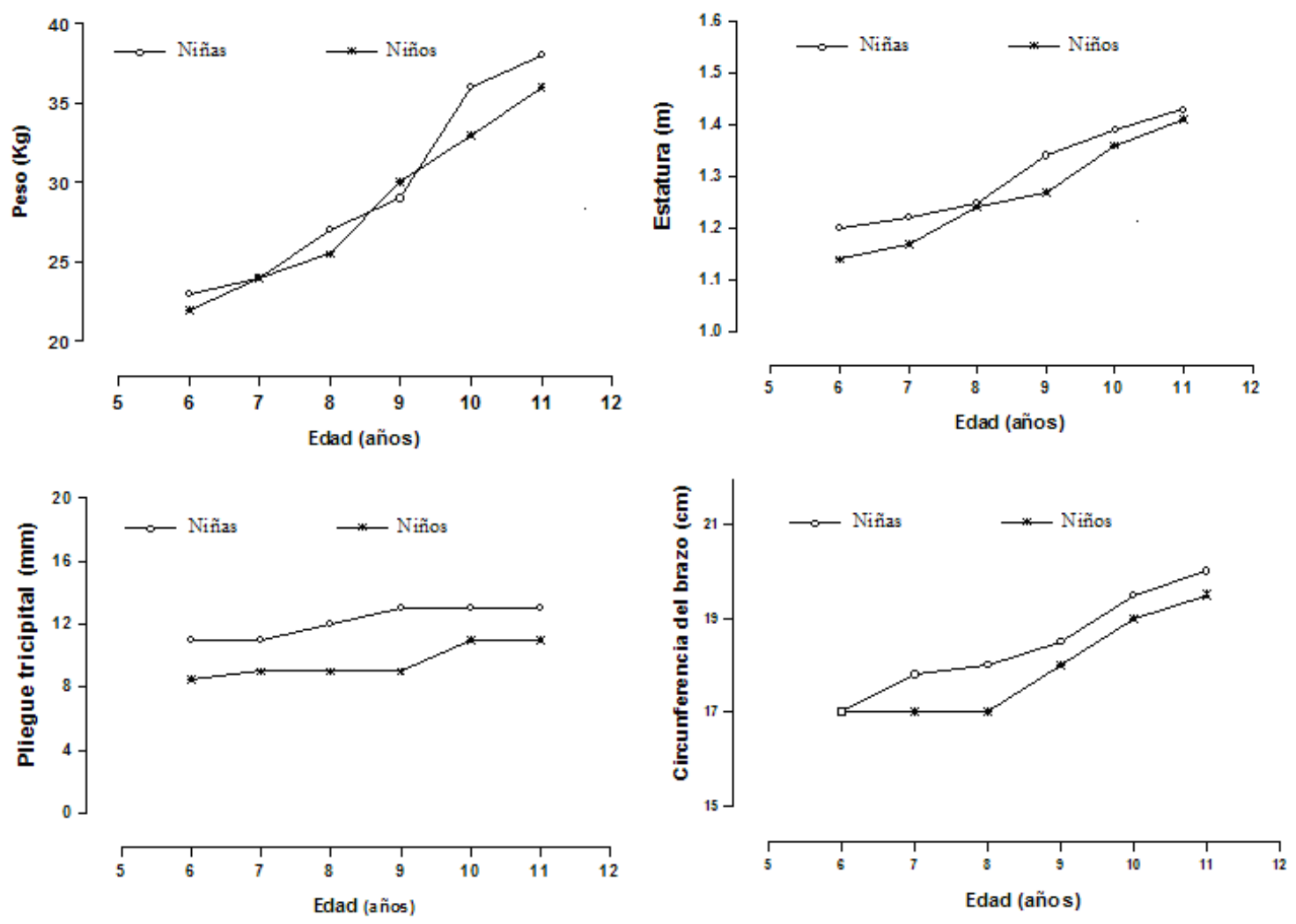

Figura 1. Valores medios del peso corporal, estatura, pliegue tricipital y circunferencia del brazo, según sexo.
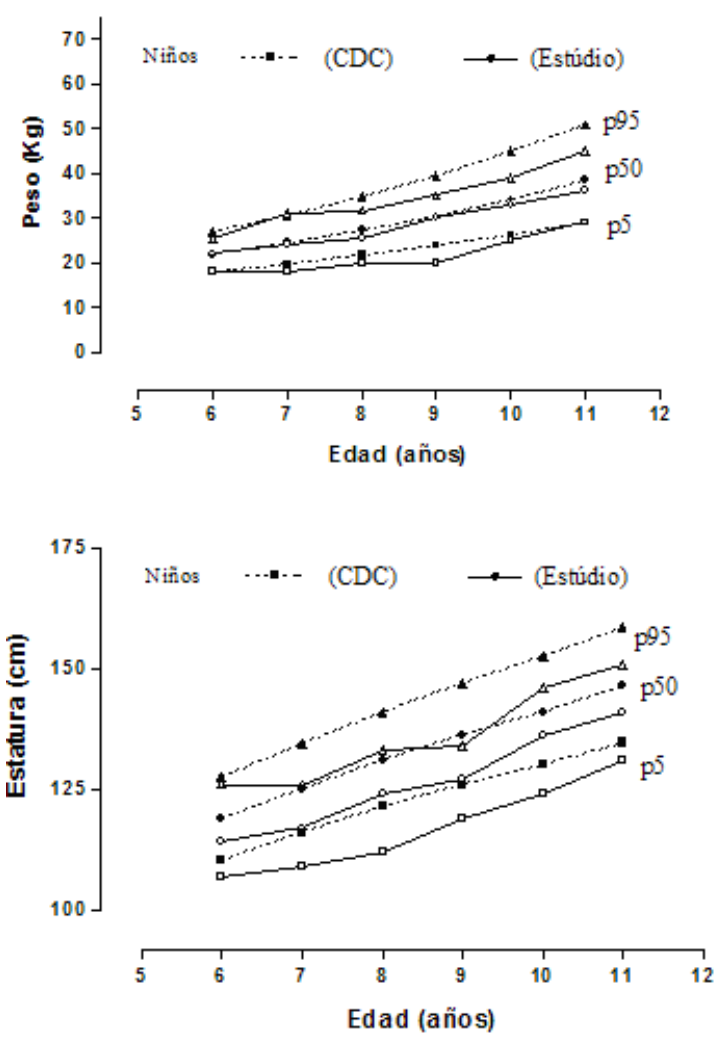
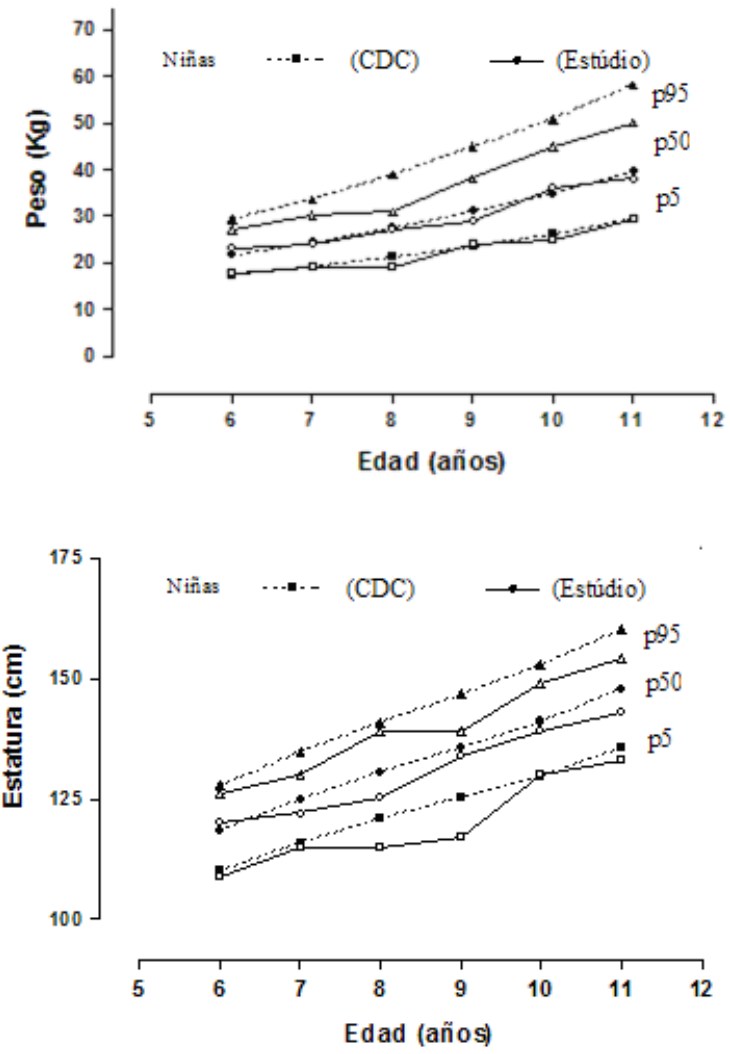

Figura 2. Comparación del peso corporal y estatura entre la referencia CDC y el estudio (p5, p50 y p95), por sexo. 
Tabla 4. Diferencias del pliegue tricipital $(\mathrm{mm})$ entre la referencia NHANAS-USA y el estudio, según percentiles.

\begin{tabular}{lccccccc}
\hline Edad & $\mathbf{P 5}^{\circ}$ & $\mathbf{P 1 0}^{\circ}$ & $\mathbf{P 2 5}^{\circ}$ & $\mathbf{P 5 0}^{\circ}$ & $\mathbf{P 7 5}^{\circ}$ & $\mathbf{P 9 0}^{\circ}$ & $\mathbf{P 9 5}^{\circ}$ \\
\hline Niños & & & & & & & \\
$6-6,9$ & 1,7 & $-3,4$ & $-5,7$ & $-2,6$ & 0,0 & 5,3 & 9,0 \\
$7-7,9$ & 0,0 & 4,5 & 0,0 & 0,0 & 3,7 & 9,6 & 12,4 \\
$8-8,9$ & 9,6 & $-6,6$ & 0 & $-5,1$ & $-5,6$ & 0,0 & 5,7 \\
$9-9,9$ & 7,9 & 0,0 & $-5,7$ & 4,5 & 3,4 & 11,6 & 6,7 \\
$10-10,9$ & 0,0 & $-16,6$ & $-5,1$ & $-4,1$ & 4,9 & 13,4 & 13,1 \\
$11-11,9$ & $-2,4$ & $-12,4$ & $-9,6$ & 0,0 & 9,0 & 12,4 & 14,9 \\
Niñas & & & & & & & \\
$6-6,9$ & 9,6 & $-7,31$ & $-2,6$ & $-4,1$ & 0,0 & 5,5 & 9,0 \\
$7-7,9$ & 5,3 & $-10,91$ & 0,0 & 0,0 & 0,0 & 0,0 & 5,1 \\
$8-8,9$ & -14 & $-7,18$ & $-5,4$ & 0,0 & 2,9 & 7,9 & 19,6 \\
$9-9,9$ & $-5,1$ & $-4,57$ & $-7,9$ & 0,0 & 5,7 & 11,2 & 13,8 \\
$10-10,9$ & $-5,7$ & $-0,95$ & $-4,1$ & $-3,4$ & 2,6 & 15,7 & 13,6 \\
$11-11,9$ & $-5,1$ & $-4,57$ & $-7,9$ & 0,0 & 10,1 & 14,9 & 14,6 \\
\hline
\end{tabular}

Diferencia entre percentiles efectuado por: 100Log (percentil NHANAS-USA/Estudio).
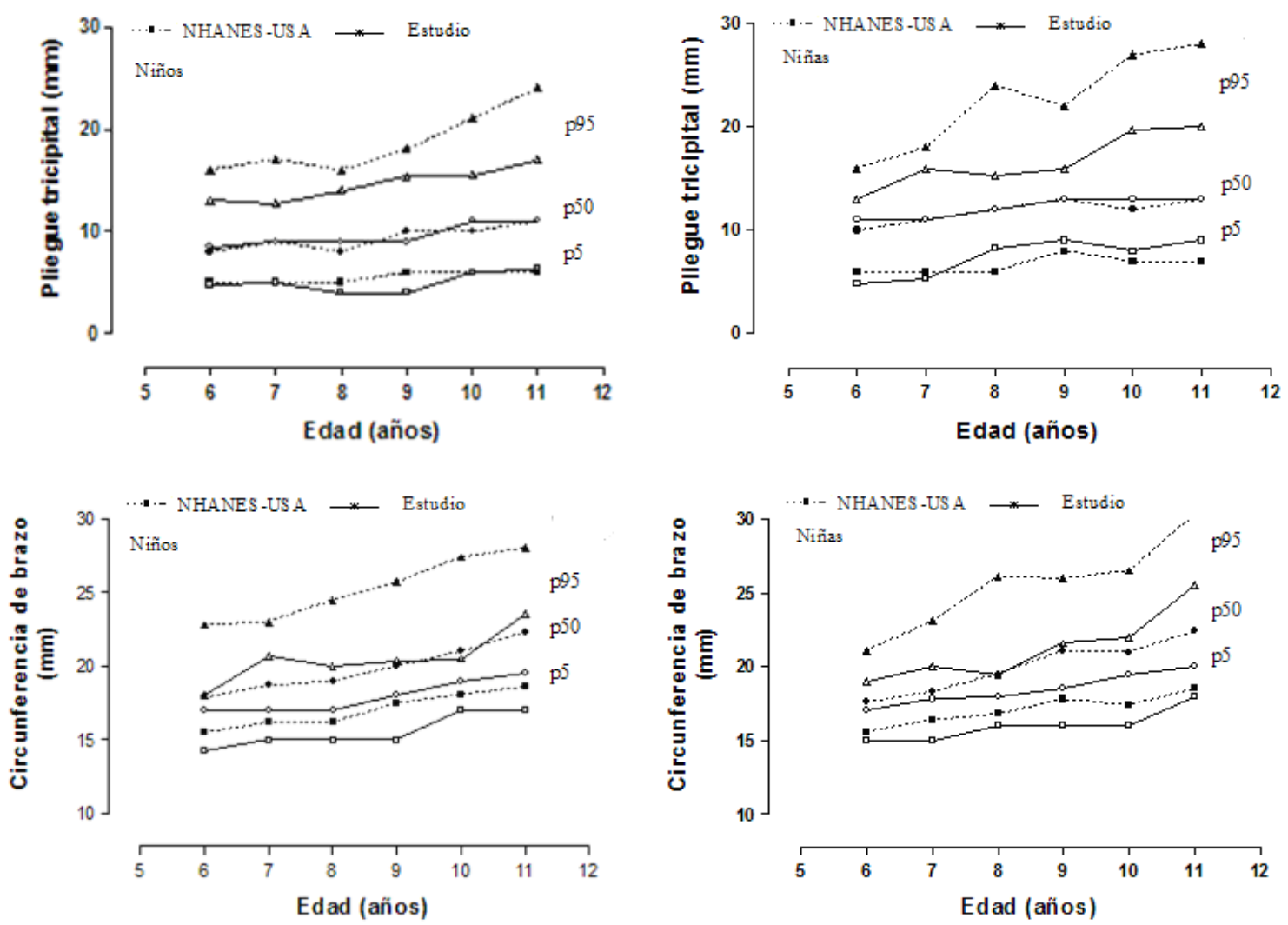

Figura 3. Comparación del pliegue tricipital y circunferencia del brazo por percentiles (p5, p50 y p95) entre la NHANAS-USA y el estudio, por sexos. 
Tabla 5. Diferencias de la circunferencia de brazo $(\mathrm{cm})$ entre la referencia NHANAS-USA y el estudio, según percentiles.

\begin{tabular}{|c|c|c|c|c|c|c|c|}
\hline & $\mathbf{P 5}^{\circ}$ & $\mathbf{P}^{\circ} 0^{\circ}$ & $\mathbf{P}^{\circ} 5^{\circ}$ & P50 ${ }^{\circ}$ & $\mathbf{P 7 5}^{\circ}$ & $\mathrm{P90}^{\circ}$ & $\mathrm{P95}^{\circ}$ \\
\hline \multicolumn{8}{|l|}{ Niños } \\
\hline $6-6,9$ & 3,5 & 2,5 & 1,8 & 2,2 & 3,3 & 6,4 & 10,2 \\
\hline $7-7,9$ & 3,3 & 1,8 & 4,3 & 4,1 & 4,7 & 7,9 & 4,5 \\
\hline $8-8,9$ & 3,3 & 2,6 & 3,0 & 4,8 & 5,0 & 4,1 & 8,8 \\
\hline $9-9,9$ & 6,6 & 1,9 & 2,8 & 4,5 & 7,6 & 9,5 & 10,0 \\
\hline $10-10,9$ & 2,7 & 0,9 & 3,3 & 4,3 & 7,3 & 11,5 & 12,6 \\
\hline $11-11,9$ & 3,9 & 2,3 & 3,8 & 5,8 & 7,0 & 5,3 & 7,6 \\
\hline \multicolumn{8}{|l|}{ Niñas } \\
\hline $6-6,9$ & 1,4 & $-0,2$ & 1,8 & 2,2 & 1,8 & 6,3 & 7,9 \\
\hline $7-7,9$ & 3,3 & 2,4 & 4,3 & 2,1 & 4,7 & 6,9 & 6,0 \\
\hline $8-8,9$ & 0,5 & 1,2 & 2,2 & 2,3 & 3,9 & 6,3 & 9,9 \\
\hline $9-9,9$ & 3,8 & 2,1 & 4,1 & 3,3 & 2,6 & 6,5 & 7,5 \\
\hline $10-10,9$ & 5,3 & 3,4 & 4,9 & 3,2 & 4,1 & 8,2 & 9,5 \\
\hline $11-11,9$ & 1,4 & 1,7 & 2,6 & 4,7 & 6,5 & 1,8 & 4,0 \\
\hline
\end{tabular}

Diferencia entre percentiles efectuado por: 100 Log (percentil NHANAS-USA/Estudio).

los valores del pliegue tricipital de la referencia NHANAS-USA y el estudio, de acuerdo a los percentiles. Se observa valores similares desde el p5 hasta el p50, luego, a parir del p75, p90 y p95 los valores de la referencia muestran valores superiores (Figura 3).

La diferencia entre los valores de la circunferencia del brazo de la referencia NHANAS-USA y el estudio, se puede observar en la tabla 5. Se encontró que en todas las edades y en todos los percentiles, los valores de la referencia son más altos (Figura 3).

\section{DISCUSIÓN}

Las curvas de referencia representan un "modelo empírico saludable" y sirven en un solo tiempo para clasificar y diagnosticar el estado nutricional de un individuo o una población (18). Estos modelos referenciales fueron desarrollados en poblaciones del nivel del mar (11), utilizando como criterio para clasificar el estado nutricional, inclusive en poblaciones de altitud, a pesar de que varios estudios sostienen que el nivel de impacto en poblaciones de altitud es severo (3-6).

Las medidas antropométricas tradicionales de peso corporal y estatura encontradas en el estudio evidencian un crecimiento lineal con la edad, en ambos géneros, desde los 6 hasta los 11 años. Estos hallazgos sugieren patrones de crecimiento similares a los descritos por la literatura (19-21), salvo una ligera superioridad en estatura por parte de las niñas.

Comparado con niños que viven en elevadas altitudes del estudio de Marquirí y Ñuñoa (5), los niños de nuestro estudio muestran valores superiores en peso y estatura. Esto podría ser atribuido a factores como la altitud y a las mejores condiciones socioeconómicas que presentan los niños de Arequipa. Sin embargo, 
los niños de Tintaya (4100 msnm) (5) que viven en una pequeña zona urbana muestran valores similares de peso y estatura a los de escolares de Arequipa. Crespo et al (22), encontraron en escolares de altitud moderada en el Perú valores similares de peso y estatura, que nuestro estudio. Estos hallazgos nos permiten destacar que los niños que viven en regiones de altitud y en zonas urbanas muestran similar patrón de crecimiento, aunque es necesario realizar más estudios y evaluar mayor número de variables antropométricas para caracterizar de mejor forma a los escolares de moderada y elevadas altitudes.

Cuando se compararan los valores medios, con la referencia internacional del CDC (16), verificamos que tanto niños y niñas muestran valores similares en el peso corporal; pero en estatura, los niños de nuestro estudio tuvieron valores medios inferiores, lo que demuestra que los niños de moderada altitud de alguna forma muestran cierto grado de retraso en el crecimiento lineal, a pesar de tener condición socioeconómica media y vivir en zonas urbanas. Sin embargo, pareciera que el factor hipoxia podría estar involucrado ya a moderada altitud, a pesar de que la teoría define el nivel critico de respuesta compensatoria en $2500 \mathrm{msnm}$ (23) y $3000 \mathrm{msnm}$ (24), respectivamente.

Los resultados del estudio muestran un aumento del tejido adiposo en el pliegue tricipital de forma ascendente con la edad. Las niñas muestran una ligera mayor tendencia al aumento del tejido adiposo que los niños, lo que coincide con lo descrito en la literatura (19-21). Pero, cuando se compara con los niños de ambos géneros del estudio de Marquirí (5), estos muestran valores relativamente inferiores de tejido adiposo en el pliegue tricipital, pero similares a los niños de Tintaya que viven a $4100 \mathrm{msnm}$. Estos resultados sugieren que independientemente de la altitud, los niños que viven en zonas urbanas muestran similar patrón de crecimiento en el pliegue tricipital.
Sin embargo, es necesario realizar más estudios en escolares de moderada y elevada altitudes de zonas urbanas con el propósito de corroborar o rechazar los resultados obtenidos en nuestro estudio.

Cuando se comparó el pliegue tricipital con la referencia NHANAS-USA para niños blancos caucásicos descrita por Frisancho (17), los resultados fueron similares en el p5, p25 y p50; pero en el p75, p90 y p95, fueron menores a los de la referencia; la figura 3 ilustra claramente esta tendencia en niños de ambos géneros. Esto indica que los escolares de moderada altitud no llegan a mostrar altos valores de tejido adiposo, consecuentemente no se observa sobrepeso y obesidad respecto al pliegue tricipital en ambos géneros.

La circunferencia del brazo es ampliamente utilizada en estudios de crecimiento y desarrollo (25-27), Los resultados de nuestro estudio fueron similares a los descritos en la literatura (19-21). Sin embargo, hay que mencionar que no hemos encontrado estudios en escolares que viven en moderada y elevada altitud, que permitan contrastar nuestros resultados.

Los valores medios y de los percentiles de los niños de nuestro estudio fueron inferiores que los de la referencia internacional, sugiriendo que los niños de zonas urbanas de Arequipa que viven a moderada altitud muestran retraso en el crecimiento, a pesar de ser de condición socioeconómica media.

Este estudio es uno de los primeros trabajos que muestra los parámetros del crecimiento físico de niños escolares que viven a moderada altitud, por lo que es necesario realizar más estudios ampliando los grupos etarios y determinar los parámetros del crecimiento físico en función de otras referencias internacionales, así como caracterizar a los escolares no sólo a partir de variables de crecimiento, sino también de la composición corporal. Es necesario resaltar 
que en nuestro estudio no se controló el tipo de alimentación, ni el nivel de actividad física, lo que hubiera permitido discutir e interpretar de mejor forma los resultados obtenidos. Por lo tanto, los resultados de este estudio deben ser tomados con cautela y precaución.

Se concluye que los escolares que viven a moderada altitud muestran valores de peso corporal y pliegue tricipital similares a los de las referencias internacionales, y menores valores de estatura y circunferencia del brazo, por lo que los resultados sugieren atraso en el crecimiento lineal que podría estar relacionada con la altitud y con una pobre reserva de proteína observada en la circunferencia del brazo.

\section{Correspondencia:}

\section{Marco Antonio Cossio-Bolaños \\ Av. Erico Veríssimo 701. Cidade Universitaria, CEP, 13083-851 \\ Campinas, SP; Brasil. \\ Correo electrónico mcossio30@hotmail.com}

\section{REFERENCIAS BIBLIOGRÁFICAS}

1. Marcondes E. Desenvolvimento da criança: Desenvolvimento biológico crescimento. Rio de Janeiro: Sociedade Brasileira de Pediatria; 1994. p. 75.

2. Nahas MV, Corbin NC. Educação para aptidão física e saúde: Justificativa e sugestões para implantação nos programas de educação física. Rev bras ciênc mov. 1992; 5(3): 14-24.

3. Leonard WR, Dewalt K, Stansbury P, and Mcaston $\mathrm{K}$. Growth differences between children of highland and Coastal Ecuador. Am j phys anthropol. 1995; 98: 47-57.

4. Lawrence P, Haas JD. Physical growth and maximal work capacity in preadolescent boys at high-altitude. Hum biol. 1982; 5(4): 677-695.

5. Pawson I, Huicho L, Muro M, Pacheco A. Growth of children in two economically diverse Peruvian high-altitude communities. Am J hum biol. 2001; 13: 323-340.

6. Cossio-Bolaños MA, Arruda M, Gómez R. Crecimiento físico en niños de 6 a 12 años de media altura de Arequipa - Perú (2320msnm). Rev Inter Ciencias Deporte. 2009; 14(5): 32-44.
7. Zemel BS, Riley EM, Stallings VA. Evaluation of methodology for nutritional assessment in children: anthropometry, body composition, and energy expenditure. Ann Rev Nutr. 1997; 17: 211-235.

8. Gharib NM, Shah P. Anthropometry and body composition of school children in Bahrain. Ann Saudi Med. 2009: 29: 258-269.

9. Arruda M. Factores de crecimiento físico y aptitud física en pre-escolares. Rev bras ciênc mov. 1993; 1(1): 73-82.

10. Onis M, Habicht JD. Anthropometric reference data for international use; recomendations from a world health organization expert committee. Am J Clin Nutr. 1996; 64:650-658.

11. Cossio-Bolaños MA, Arruda M, Gómez RA. Características antropométricas de niños $\mathrm{y}$ adolescentes de altura. In: Salazar CM. (Editor). Usos y representaciones de las prácticas físicas-deportivas de los jóvenes mexicanos. Primera edición. México DF: Universidad Colima y Veracruzana; 2010.p. 185199.

12. Ponce de León S. The WHO Multicentre growth reference study and altitude above sea level. An example of hyposometric bias. High Alt Med Biol. 2008; 9:3.

13. González F. Metabolismo en las grandes alturas. Acta andin. 2001; 9(1-2):31-42.

14. Cossio-Bolaños MA. Crescimento físico e desempenho motor em crianças de 6-12 anos de condição socioeconômica média da área urbana de Arequipa (Perú). Dissertação de mestrado. Facultad de Educación Física de la Universidad Estatal de Campinas. Sao Paulo, Brasil, 2004.

15. Ross WD y Marfell-Jones MJ. Kinanthropometry. En: MacDougall JD, H.A, Wenger HA, Geen HJ (Eds). Physiological testing of elite athlete. London: Human Kinetics; 1991. p. 223-308.

16. Center for disease control and preventive, National Center for Health Statistics. CDC, growth charts: United States, 2002. URL disponible en: http:/www. ede.gov./growthcharts (Fecha de acceso: enero 2012).

17. Frisancho AR. New norms of upper limb fat and muscle areas for assessment of nutritional status. Am J Clin Nutr. 1981; 34: 25-40.

18. Conde W, Monteiro CA. Body mass index cutoff points for evaluation of nutritional status in Brazilian children and adolescents. J Pediatr (Rio J). 2006; 82 (4): 266-72.

19. Malina RM, Bouchard C. Growth maturation and physical activity. Champaign, Il: Human Kinetics; 1991.

20. Forbes GB. Human body composition: Growth, aging, nutrition and activity. New York: Springer Verlag; 1987.

21. Guedes DP, Guedes JERP. Crescimento, composição corporal e desempenho motor de crianças. Sao Paulo: 
CRL Balieiro; 1997.

22. Crespo I, Valera J, Gonzalez G, Guerra-Garcia R. Crecimiento y desarrollo de niños y adolescentes a diversas alturas sobre el nivel del mar. Acta andin. 1995; 4(1):53-64.

23. Ramirez A. Exposición toxicológica en las grandes alturas: ¿Es necesario corregir los valores límite umbral de exposición de tóxicos? An Fac Med. 2011; 72(1): 61-7.

24. Hackett PH, Oelz O. The Lake Louise Consensus on the definition and quantification of altitude illness. En: Sutton JR, Coates G, Houston CS. Eds. Hypoxia and Mountain Medicine. Burlington. USA: Queen City
Printers. 1992.

25. Cossio-Bolaños MA, Arruda, Marco A. Correlación entre el índice de masa corporal y las circunferencias corporales de niños de 4 a 10 años. An Fac Med. 2010; 71 (2):79-82.

26. Malina RM, Bouchard C, Bar-Or C. Growth maturation and physical activity. $2^{\mathrm{a}}$ ed. Champaign,

Recibido: 16/09/10

Aceptado para publicación: 29/03/12 